\title{
Thermoelectricity in Quantum Hall Corbino Structures
}

\author{
Mariano Realø, ${ }^{1, *}$ Daniel Gresta, ${ }^{2}$ Christian Reichl,${ }^{3}$ Jürgen Weis, ${ }^{4}$ Alejandra Tonina, ${ }^{1}$ Paula Giudici, ${ }^{5}$ \\ Liliana Arrachea, ${ }^{2}$ Werner Wegscheider, ${ }^{3}$ and Werner Dietsche ${ }^{3,4}$ \\ ${ }^{1}$ Instituto Nacional de Tecnología Industrial, INTI and INCALIN-UNSAM, Avenida General Paz 5445, \\ Buenos Aires 1650, Argentina \\ ${ }^{2}$ International Center for Advanced Studies, ECyT-UNSAM, 25 de Mayo y Francia, Buenos Aires 1650, Argentina \\ ${ }^{3}$ Solid State Physics Laboratory, ETH Zürich, Zürich CH-8093, Switzerland \\ ${ }^{4}$ Max-Plack-Institut für Festkörperforschung, Heisenbergstrasse 1, Stuttgart D-70569 Germany \\ ${ }^{5}$ INN CNEA-CONICET, Avenida General Paz 1499, Buenos Aires 1650, Argentina
}

(Received 11 March 2020; revised 10 August 2020; accepted 14 August 2020; published 8 September 2020)

\begin{abstract}
We measure the thermoelectric response of Corbino structures in the quantum Hall effect regime and compare it with a theoretical analysis. The measured thermoelectric voltages are qualitatively and quantitatively simulated based upon the independent measurement of the conductivity, indicating that they originate predominantly from the electron diffusion. In contrast to earlier Hall-bar experiments, electronphonon interaction does not lead to a phonon-drag contribution. This implies a description of the Onsager coefficients on the basis of a single transmission function, from which both thermovoltage and conductivity can be predicted with a single fitting parameter. Furthermore, it lets us predict a figure of merit for the efficiency of thermoelectric cooling, which becomes very large for partially filled Landau levels and high magnetic fields.
\end{abstract}

DOI: 10.1103/PhysRevApplied.14.034019

\section{INTRODUCTION}

The quantum Hall effect (QHE), which occurs in twodimensional electron systems (2DES) exposed to quantizing magnetic fields, is one of the most prominent examples of the synergy between fundamental physics and quantum technologies [1]. It is topological in nature and intrinsically related to exotic properties of matter, such as fractionalization and non-Abelian statistics [2-4]. At the same time, these complex properties are precisely the reason for its robustness and appeal for practical applications. It is nowadays at the heart of the definition of the electrical metrological standards [5], while it is also a promising platform for the development of topological quantum computation [6].

Measurements of the entropy would be of great importance in verifying the theoretically expected quantum states, particularly of the non-Abelian ones. One possibility to access entropy in a 2DES is to measure the thermoelectricity $[7,8]$. However, although thermoelectricity has been studied both experimentally and theoretically since the discovery of the integer QHE [9-11], it has not been possible to reconcile the experimental results obtained with Hall bars [Fig. 1(a)] with theories based upon electron diffusion. The overwhelming effect of phonon drag has been

\footnotetext{
*mreal@inti.gob.ar
}

invoked as one reason $[9,10,12]$ but, more recently, inherent problems connected with the topology of the Hall-bar geometry, affecting both phonon drag and electron diffusion, have been realized [8]. The longitudinal thermopower (or Seebeck coefficient) measured along a Hall bar closely resembles the longitudinal resistance, both in the phonon drag and in the diffusive regime, while an oscillating behavior with sign changes is expected by the theory. It has been suggested that the longitudinal thermopower could be measured correctly in Corbino geometry [Fig. 1(b)] where, due to the circular geometry, the thermal bias is applied radially; hence any thermal and electrical transport is induced along the radial direction [13] and the transport takes place through the bulk.

Early thermopower experiments in Corbino geometry have failed to observe the expected sign-changing behavior [14]. It has been reported, however, in other experimental works using the Corbino geometry [15]. The latter have used rf heating of the 2DES to produce the temperature gradient directly in the 2DES, claiming that no phonons are involved in the measured thermopower.

In this paper, we report Corbino thermopower measurements in the QHE regime by using a conventional heater in the center of the device. In this way, a temperature gradient is set up in both the substrate and the 2DES. Results at temperatures from about $300 \mathrm{mK}$ to $2 \mathrm{~K}$ are presented and compared with theoretical results, where both the electrical 

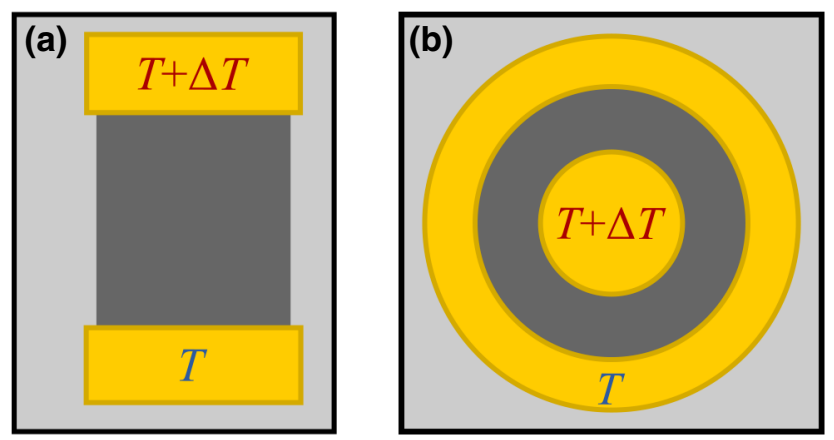

FIG. 1. The two sample designs to investigate thermoelectric effects: (a) the Hall bar; (b) the Corbino. The dark-gray areas are the 2DES. The hot and the cold contacts for measuring the thermovoltage are at the two ends of the rectangular-shaped Hall bar. For the Corbino, the hot contact is in the center of the 2DES, which is surrounded by the cold contact.

conductance and the thermopower are modeled based upon the same transmission function. A very good agreement over our range of magnetic fields and temperatures is found. This demonstrates that the substantial disagreement that is typical for Hall bars can be removed by using the Corbino topology. In contrast to Hall-bar studies, it is not necessary to consider phonon drag in the theory.

\section{EXPERIMENTAL DETAILS}

\section{A. Setup}

Our setup is sketched in Fig. 2. An Au-Pd thin-film heater is inserted in the center of the Corbino samples and heated with an ac current with a frequency $f$ of a few hertz, producing a temperature oscillation of $2 f$. In this way, a radial thermal gradient is induced between the center and the external edge of the sample, which is assumed to be close to the temperature $T$ of the bath. The device used here consists of five concentric ohmic contact rings with diameters ranging from $0.4 \mathrm{~mm}$ to $3.2 \mathrm{~mm}$, made by alloying $\mathrm{Au}-\mathrm{Ge}-\mathrm{Ni}$ into the 2DES structure to form four independent Corbino rings. Under the heater and outside of the rings, the 2DES is removed. It is assumed that the local temperature over the 2DES follows that of the underlying GaAs substrate. This has already been verified by Chickering et al. [16,17] down to much lower temperatures than the ones used here. We neglect possible anisotropies in the heat conductivity of the substrate due to the ballistic nature of the phonons, because these become only relevant if the dimensions of the heater and the contacts are much smaller than the substrate thickness [18].

The four Corbino rings of this device allow us not only to measure the thermopower at four different radial distances from the heater but also to determine, in a different experiment, the temperatures at the different ring positions. This can be done by using the conductances of the four (a)

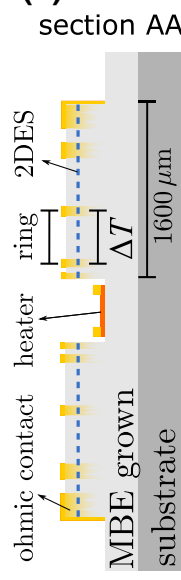

(b)

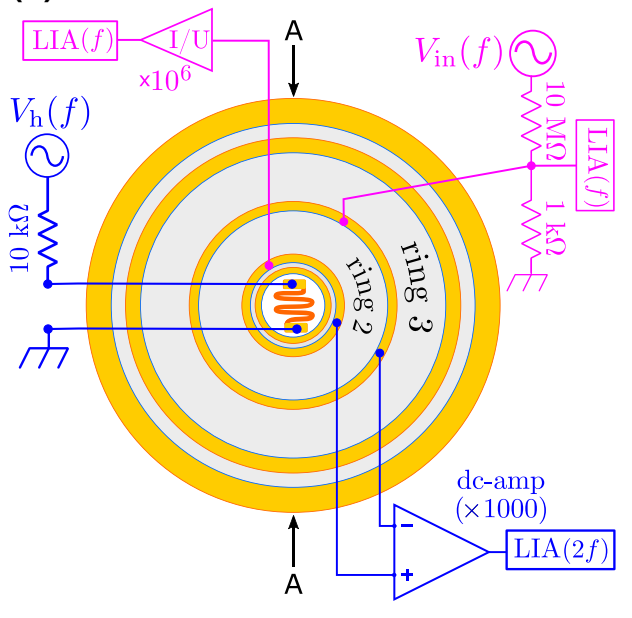

FIG. 2. The scheme of the experimental setup. (a) A cross section of the sample: note that the heater element is over the substrate outside the 2DES. (b) The measurement configurations for the conductance and the thermovoltage are shown in magenta and blue, respectively. "LIA" denotes the lock-in amplifier. The two types of experiment are done in separate runs. Only two of the four Corbino rings are labeled in the figure.

Corbinos as thermometers. Measurement of the conductance as a function of the bath temperature without any heat applied is used for calibration. With the heater on, the temperatures at the different rings can be measured.

As a response to the thermal bias between the center and the edge of the sample, charges diffuse across the Corbino ring, which is compensated by generating a voltage with frequency $2 f$ between the inner and the outer circumferences. The sign and the magnitude of this thermovoltage are determined by the transmission function, as discussed in the theory section. The thermoelectric response in this device is much simpler than the one in the Hall-bar geometry, where the transport takes place along longitudinal and transverse directions with respect to the applied biases. In fact, in the Corbino geometry, the thermovoltage develops along the direction of the temperature bias.

The samples are grown by molecular-beam epitaxy on GaAs wafers having a single 2DES located in a 30-nm-wide quantum well with Si-doped layers on both sides. Data from two samples from two wafers, $A$ and $B$, are presented here. Separate test pieces from these wafers in van der Pauw geometry have mobilities of $21 \times 10^{6} \mathrm{~cm}^{2} \mathrm{~V}^{-1} \mathrm{~s}^{-1}$ and $18 \times 10^{6} \mathrm{~cm}^{2} \mathrm{~V}^{-1} \mathrm{~s}^{-1}$ at electron densities of $n_{e}=3.06 \times 10^{11} \mathrm{~cm}^{-2}$ and $n_{e}=2.0 \times$ $10^{11} \mathrm{~cm}^{-2}$, respectively, measured at $1.3 \mathrm{~K}$ in the dark.

The Corbino samples are glued in a standard commercial ceramic holder with a gold-plated base and pins and a 3-mm-diameter hole drilled in the middle to reduce thermal contact with the samples. The measurements are performed in vacuum in a ${ }^{3} \mathrm{He}$ cryostat equipped with a 
14-T magnet, with the ability to achieve a base temperature of $250 \mathrm{mK}$.

Figure 2 also shows the configurations used for the measurements of the conductance (magenta) and of the thermovoltage (blue). The conductance $G$ is measured by applying an ac voltage through a voltage divider and measuring the current with an amplifier (IUAmp). The thermovoltage $V_{\text {tp }}$ is measured in separate runs by passing an ac current of frequency $f$ to the central heater, which has a resistance of about $650 \Omega$. The thermopower induced in the sample is measured by using a $\times 1000$ differential dc voltage amplifier (dc-amp). The input impedance of this amplifier must be very high, because the internal resistance of the Corbino device diverges in the quantum Hall states. We use an amplifier with an input impedance of about $1 \mathrm{~T} \omega$ [19]. Very little frequency dependence of the thermovoltage is found between $f=3 \mathrm{~Hz}$ and $100 \mathrm{~Hz}$. Most measurements are done at $f=13.8 \mathrm{~Hz}$. To avoid effects of time-dependent magnetic fluxes, the waiting time for each data point is set to a few seconds to guarantee the stabilization of the magnetic fields at a constant value.

\section{B. Thermovoltage measurement}

The experimental results for both the thermal voltage (solid blue) and the conductance (solid orange) of sample A are shown in Fig. 3 at a base temperature $T$ of $269 \mathrm{mK}$ and an average heater power $P$ of $277 \mathrm{nW}$. The magnetic field is swept from 0.3 to $5 \mathrm{~T}$. The conductance shows the typical Shubnikov-de-Haas $(\mathrm{SdH})$ oscillations, with the spin splitting becoming visible at about $0.9 \mathrm{~T}$ and the conductance minima approaching zero even at filling factors less than 20. The thermovoltage $V_{\text {tp }}$ shows numerous features. At small magnetic fields, it oscillates with a similar periodicity as the conductance, changing sign at both the conductance maxima and minima. At higher magnetic fields, additional features appear in the regions of the conductance minima, which become very significant and chaotic at even larger magnetic fields, where the conductance minima are wider. Between the conductance minima, the thermovoltage $V_{\text {tp }}$ now changes to a sawtoothlike behavior, still changing sign at both the maxima and the minima of the conductance. Such sign changes have not been observed in the earlier Hall-bar experiments but have already been seen in the previous rf-based Corbino experiments [15] and have been expected theoretically [8].

We observe the sign-change behavior in a similar way on several samples with different densities and mobilities. The data for sample $B$ are presented in Fig. 4, showing $V_{\text {tp }}$ measured across the three different outer Corbino rings at a temperature of $600 \mathrm{mK}$. The oscillatory behavior of $V_{\mathrm{tp}}$ is again clearly visible, as are the large signals in the regions that correspond to the conductance minima.

The large signals have not been reported before. These are not spurious signals: they are reproducible and they

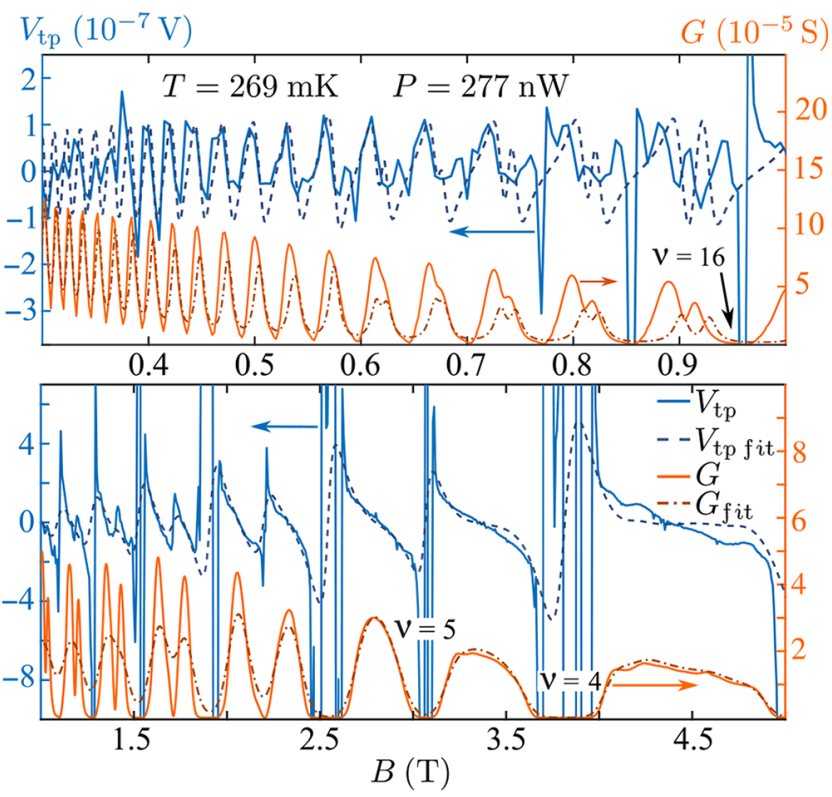

FIG. 3. The conductance $G$ and thermovoltage $V_{\text {tp }}$ as a function of the magnetic field $B$ for ring 2 in Fig. 2 at temperature $T$ with power $P$ supplied at the heater. The experimental data are plotted using solid lines. The theoretical (dashed) plots are based on the calculation of Eq. (2), with the respective inferred transmission functions.

persist if the magnetic field is stopped and kept constant or if the sweep direction is reversed. They are definitely thermally induced signals and are not produced by an electromagnetic crosstalk. The large signals vanish by applying a dc current on top of a square ac current. This leads to a constant heating and thus a vanishing temperature oscillation but it would leave any suspected crosstalk unchanged. We will speculate at the end of this paper about the possible origins of the large signals.

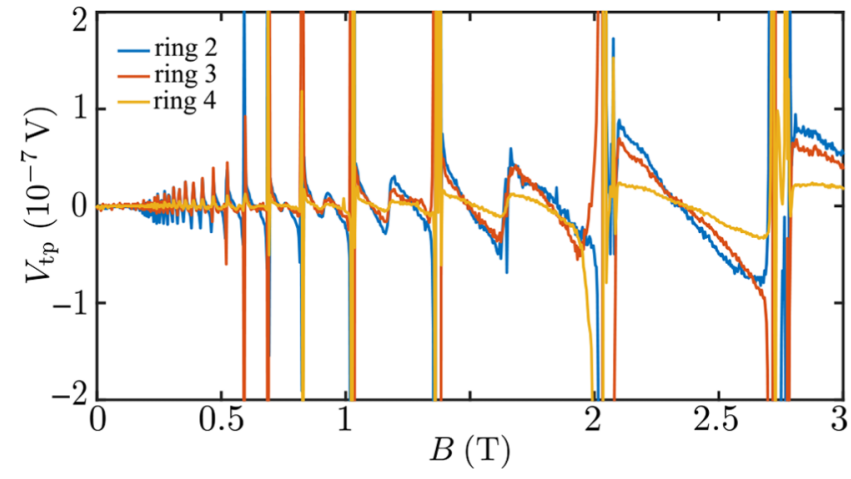

FIG. 4. The $V_{\text {tp }}$ response of sample $B$ at different rings at a bath temperature of $600 \mathrm{mK}$ and a heater power of $213 \mathrm{nW}$. Rings 2 and 3 present a greater temperature gradient and hence a larger voltage response than ring 4. 
In the following, we concentrate on the analysis of the thermal voltages outside of the SdH minima. We show that the magnetic field traces of both $V_{\mathrm{tp}}$ and the conductance $G$ can be fitted using only charge diffusion. The same transmission function based upon model Landau levels (LLs) is used for calculating both the conductance and the thermovoltage. The only fitting parameter is the temperature gradient. The resulting fits are already shown in Fig. 3 as dashed lines.

\section{THERMOELECTRIC RESPONSE}

\section{A. Onsager coefficients}

To describe the thermoelectric transport, we consider the Corbino geometry in Fig. 1(b). The Corbino ring acts as a conductor in the radial direction between the hot and cold reservoirs, with a temperature bias of $\Delta T$. In the linear response, the corresponding charge and heat currents for small $\Delta T$ and bias voltage $V$ can be expressed as follows [20]:

$$
\left(\begin{array}{c}
I^{C} / e \\
I^{Q}
\end{array}\right)=\left(\begin{array}{cc}
\mathcal{L}_{11} & \mathcal{L}_{12} \\
\mathcal{L}_{21} & \mathcal{L}_{22}
\end{array}\right)\left(\begin{array}{c}
X_{1} \\
X_{2}
\end{array}\right)
$$

where $X_{1}=e V / k_{B} T, X_{2}=\Delta T / k_{B} T^{2}$, and $\hat{\mathcal{L}}$ is the Onsager matrix. The electrical and thermal conductances are, respectively, $G=e^{2} \mathcal{L}_{11} / T$ and $\kappa=\operatorname{Det} \hat{\mathcal{L}} /\left(T^{2} \mathcal{L}_{11}\right) . S=$ $\mathcal{L}_{12} / \mathcal{L}_{11}$ defines the Seebeck coefficient and $\Pi=\mathcal{L}_{21} / \mathcal{L}_{11}$ is the Peltier coefficient. For ballistic or diffusive transport, $\mathcal{L}_{i j}$ depends only on the quantum dynamics of the electrons in the presence of the magnetic field and the disorder of the sample. These are described by a transmission function $\mathcal{T}(\varepsilon)$,

$$
\mathcal{L}_{i j}=-T \int \frac{d \varepsilon}{h} \frac{\partial f(\varepsilon)}{\partial \varepsilon}(\varepsilon-\mu)^{i+j-2} \mathcal{T}(\varepsilon)
$$

where $f(\varepsilon)=1 /\left(e^{(\varepsilon-\mu) / k_{B} T}+1\right)$ is the Fermi distribution function, $\mu$ is the chemical potential, and $T$ is the temperature of the electrons. In the presence of disorder and the absence of electron-electron interactions, $\mathcal{T}(\varepsilon)$ has originally been calculated by Jonson and Girvin [21]. At high temperatures, electron-phonon interaction gives rise to an additional component in the transport coefficients $\mathcal{L}_{i j}$.

\section{B. Conductance and thermovoltage}

Our goal is to accurately describe the electronic component of the Onsager coefficients obtained from the experimental data. At first, we measure the conductance $G(B)$ as a function of the applied magnetic field $B$. The thermovoltage $V_{\text {tp }}$ is measured separately and corresponds to the voltage for which $I^{C}=0$ in Eq. (1),

$$
V_{\mathrm{tp}}(B)=-S(B) \frac{\Delta T}{T} .
$$

Here, $S(B)$ is the Seebeck coefficient as a function of the magnetic field, $T$ is the temperature of the bath (cold finger in our case), and $\Delta T$ is the temperature difference between the two contacts of the Corbino ring under investigation. From the data for $G(B)$, we infer the transmission function $\mathcal{T}(\varepsilon)$ entering Eq. (2). Given $\mathcal{T}(\varepsilon)$, we can evaluate the electrical component of the other Onsager coefficients; in particular, $\mathcal{L}_{12}(B)$. Through Eq. (3), this leads to a theoretical prediction for the behavior of $V_{\mathrm{tp}}(B)$ resulting from the electrical transport, which can be directly contrasted with the experimental data.

There are two regimes to be considered for the calculation of $\mathcal{T}(\varepsilon)$ : (i) At low magnetic fields, where the different LLs are not clearly resolved, we calculate the transmission function using the model introduced in Ref. [8,21]. The latter is based on a single-particle picture for the 2DES in the presence of a magnetic field and elastic scattering introduced by impurities. (ii) For higher magnetic fields, where the differently filled LLs are clearly distinguished and are separated by a gap, we use the fact that in the limit of $T \rightarrow 0$, Eq. (2) leads to $\mathcal{T}(\mu) \sim G(\mu) / e$.

\section{Transmission function}

\section{Low magnetic field}

Here, we consider the transmission function $[8,21]$

$$
\mathcal{T}(\varepsilon)=\Lambda \sum_{n, \sigma} \frac{(n+1) \omega_{c}^{2}}{8 \pi h} A_{n, \sigma}(\varepsilon) A_{n+1, \sigma}(\varepsilon),
$$

where $\Lambda$ is a geometric factor relating the conductance to the conductivity, while $A_{n, \sigma}(\varepsilon)=\operatorname{Im}\left[G_{n, \sigma}(\varepsilon)\right]$, in which $G_{n, \sigma}(\varepsilon)=\left[\varepsilon-\varepsilon_{n, \sigma}-\Sigma(\varepsilon)\right]^{-1}$ is the Green function calculated within the self-consistent Born approximation. $\varepsilon_{n, \sigma}=\hbar(n+1 / 2) \omega_{c} \pm \mu_{B} B / 2$ is the energy of the LLs, including the Zeeman splitting, with \pm corresponding, respectively, to $\sigma=\uparrow, \downarrow$. Here, $\mu_{B}$ is the Born magneton, $\omega_{c}=e B / m^{*}$ is the cyclotron frequency, $m^{*}=0.067 m_{e}$ is the effective mass of the electrons in the structure, and $m_{e}$ is the electron mass. The effect of disorder due to impurities introduces a widening $\Gamma$ in the LLs, which is accounted for by the self-energy $\Sigma(\varepsilon)=\left(\omega-\varepsilon_{L}\right) / 2-$ $i \Gamma \sqrt{1-\left(\varepsilon-\varepsilon_{L}\right)^{2} /\left(4 \Gamma^{2}\right)}$. Here, $\varepsilon_{L}$ is the energy of the LL that is closest to $\varepsilon$. This model has two fitting parameters, $\Lambda$ and $\Gamma$, which we adjust to fit the data of the conductance $G$, through Eq. (2). This model fails to reproduce $G(B)$ for high magnetic fields $(B>1 \mathrm{~T})$. Thus a different model has to be used in this regime. 


\section{High magnetic field}

For higher magnetic fields, satisfying $k_{B} T \ll \hbar \omega_{c}$, and $\Gamma \ll \hbar \omega_{c}$, we can infer the transmission function more efficiently from the behavior of the conductance within a range of magnetic fields in the neighborhood of a given filling fraction $v$. Note that in the limit of $T \rightarrow 0$, the derivative of the Fermi function entering Eq. (2) has the following behavior: $-\partial f(\varepsilon) / \partial \varepsilon \rightarrow \delta(\varepsilon-\mu)$. Therefore, for low temperatures, such that $k_{B} T \ll \hbar \omega_{c}$, we have

$$
\mathcal{T}\left(\mu_{v}\right) \sim \frac{G\left(\mu_{v}\right)}{e}, \quad \mu_{v}=\frac{\hbar e B}{2 m^{*}}, \quad B_{v+1}<B<B_{v},
$$

where $B_{v}=n_{e} h /(e v)$ is the magnetic field corresponding to the filing fraction $\nu$, while $\mu_{\nu}$ is the Fermi energy for the range of $B$ within two consecutive integer filling factors.

\section{RESULTS}

\section{A. Thermoelectric response}

The results for the conductance and the thermovoltage are shown in Fig. 3 for a temperature of $269 \mathrm{mK}$. The experimental data for $G$ and $V_{\text {tp }}$ within the lowmagnetic-field regime are shown in the upper panel of the figure along with the theoretical description based on the transmission function of Eq. (4).

In the case of a high magnetic field, shown in the lower panel, the theoretical description is based on the transmission function of Eq. 5. Given $\mathcal{T}(\varepsilon)$, we calculate the Onsager coefficients of Eq. (2) and the Seebeck coefficient $S=\mathcal{L}_{12} / \mathcal{L}_{11}$. The ratio $\Delta T / T$ is adjusted in order to fit the experimental measurements with Eq. (3). The estimates for the temperature bias are $\Delta T=1 \mathrm{mK}$ and $1.08 \mathrm{mK}$, for low and high magnetic fields, respectively. Overall - and, in particular, for high magnetic fields - the agreement between experiment and theory is excellent within the range of $B$ corresponding to a partially filled LL, for which $G \neq 0$.

Taking into account the good agreement between the experimental and theoretical estimates of the temperature difference $\Delta T$ found in the analysis of the data of Fig. 3, we now analyze the relation between the electrical power supplied at the heater and $\Delta T$. In Fig. 5, we show experimental data for the thermovoltage at a fixed temperature and different heater powers. We assume a linear dependence between these quantities. Therefore, we fit the experimental data with the same Seebeck coefficient $S(B)$ calculated for Fig. 3 and the following dependence of the temperature difference as a function of the power: $\Delta T\left(P^{\prime}\right)=P^{\prime} / P 1.08 \mathrm{mK}$, where $P^{\prime}$ is the power corresponding to the experimental data and $P$ is the power used in the data of Fig. 3. We see very good agreement between the theoretical prediction and the experimental data. In Fig. 6, we discuss the evolution of $V_{\mathrm{tp}}$ as the temperature

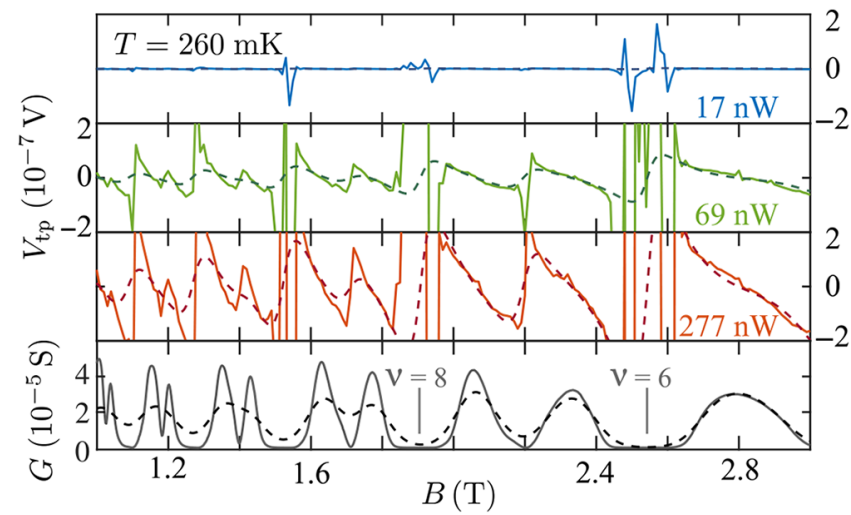

FIG. 5. The thermovoltage $V_{\text {tp }}$ for a fixed temperature and different powers $P^{\prime}$ applied at the heater, assuming $\Delta T\left(P^{\prime}\right)=$ $P^{\prime} / P 1.08 \mathrm{mK} . P$ and other details are the same as in Fig. 3 .

grows, focusing on the high-magnetic-field region. The experimental data are presented along with the theoretical prediction obtained by following the same procedure as in the previous figures and taking into account the linear dependence of $\Delta T$ with $P$ as explained in Fig. 5. Overall, the agreement between the theoretical predictions and the experimental data for magnetic fields corresponding to partially filled LLs over a wide range of temperatures is very good, improving as the temperature decreases.

\section{B. Theoretical estimate of $\Delta T$}

From the behavior of the conductance, we can infer the transmission function $\mathcal{T}(\varepsilon)$ as explained before, from which we can calculate the Onsager coefficients $\mathcal{L}_{i j}$. We recall that the thermovoltage is defined in Eq. (3). Given the calculation of $S(B)$, we need to adjust the parameter $\Delta T / T$ in order to fit the data. Since the latter enters as the slope in the linear function $V(S)$, we analyze plots of the measured $V_{\text {tp }}$ versus the calculated $S$ for values of $B$ within which the LLs are partially filled and we fit a linear function to obtain the slope. Examples are shown in Fig. 7 for two different LLs, with bath temperature $T=269 \mathrm{mK}$ and a power of $277 \mathrm{nW}$.

The corresponding fits cast $\Delta T=(1.01 \pm 0.06) \mathrm{mK}$ in the region from $B=2.21 \mathrm{~T}$ to $2.46 \mathrm{~T}$ (upper panel) and $\Delta T=(1.33 \pm 0.06) \mathrm{mK}$ in the region from $B=2.625 \mathrm{~T}$ to $3 \mathrm{~T}$ (lower panel), with uncertainties corresponding to a $95 \%$ confidence probability. Note also that the intercept, which is taken as a free parameter of the regression, is zero within the error in both cases.

The data for the other sample measured at $600 \mathrm{mK}$, shown in Fig. 4, can be analyzed similarly for several of the rings. For ring 2 , we obtain $\Delta T=(60 \pm 3) \mu \mathrm{K}$, while for ring 3 we obtain $(\Delta T=(110 \pm 10) \mu \mathrm{K}$ and for ring $4 \Delta T=(74 \pm 50) \mu \mathrm{K}$. These values are considerably lower those of sample A measured at $269 \mathrm{mK}$. The reason for this is that the thermal conductivity of the substrate 


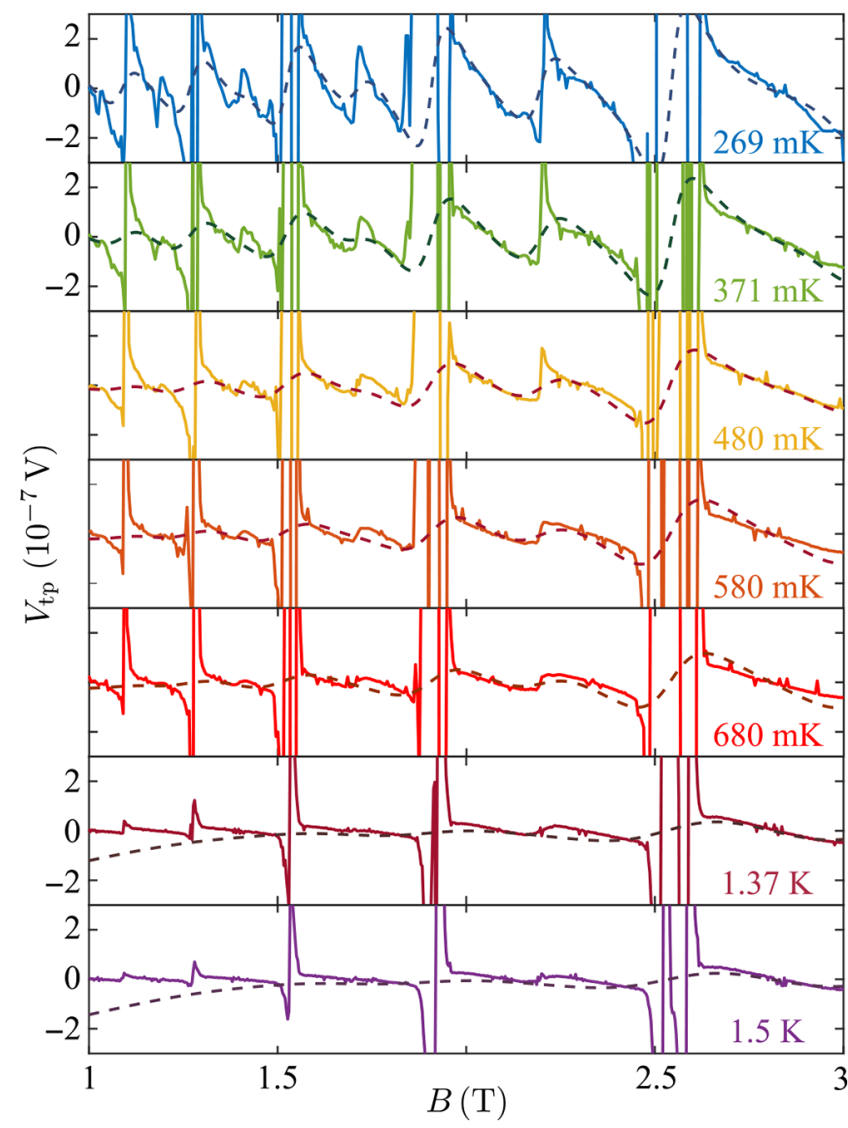

FIG. 6. The thermovoltage $V_{\mathrm{tp}}$, as function of the magnetic field for different temperatures. In the case of $269-680 \mathrm{mK}$, a power of $277 \mathrm{nW}$ is used, while for $1.37-1.5 \mathrm{~K}$, the heater power is $433 \mathrm{nW}$. The other details are the same as in previous figures. The scale for $V_{\mathrm{tp}}$ is the same in all panels.

increases with $T^{3}$ and at $600 \mathrm{mK}$ the temperature gradient will be nearly 10 times smaller and, correspondingly, a much smaller temperature difference is expected at the higher temperature.

\section{Experimental estimate of $\boldsymbol{\Delta} \boldsymbol{T}$}

The exact determination of $\Delta T$ in a Corbino device turns out to be challenging. The reason for this is that the high phonon-heat conductivity in the GaAs substrate leads to small temperature gradients between the center and the edges of the sample. One consequence is that the thermal resistance from the sample to the ceramic carrier can no longer be neglected. Using the temperature-dependent conductances of the Corbino rings as thermometers, we are nevertheless able to make estimates. The innermost and the outermost rings in Fig. 2 are used for this measurement. The conductance minimum at filling factor 9 in Fig. 3 is used because it shows a pronounced temperature dependence. The actual temperatures at these rings are found by comparing the respective conductances with

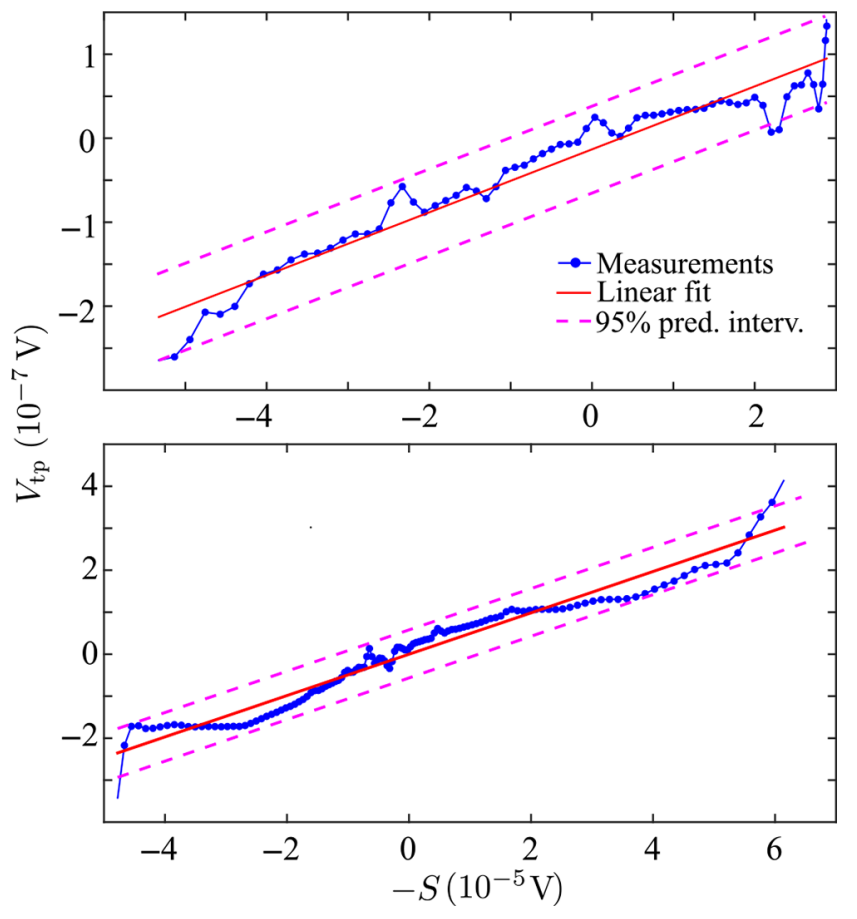

FIG. 7. The measured $V_{\text {tp }}$ signal versus the calculated $-S$ within the range of fields $B=2.21-2.46 \mathrm{~T}$ (upper panel) and $B=$ 2.625-3 T (lower panel). The slope of this relation is $\Delta T / T .95 \%$ Prediction Intervals (pred. inter.) indicates the range corresponding to $95 \%$ of reliability of the mean squares regression used in the linear fit.

the heater on and off. The temperature rise at both rings could be close to $50 \mathrm{mK}$ with the cryostat at $269 \mathrm{mK}$ and heater powers reaching $300 \mathrm{nW}$. The temperature difference between the two rings is found to be less than 9 $\mathrm{mK}$ at the highest power. This value is only an estimate because the precision and the reproducibility of the calibration procedure is limited by the temperature control of the cryostat.

The determination of $\Delta T$ could be improved by thinning the sample and thereby decreasing its thermal conductance. If this is done for several samples, it can be shown that the thermovoltage data as a function of the magnetic field becomes erratic. We suspect that the thinning leads to inhomogeneities in the 2DES, making the $V_{\text {tp }}$ measurements useless. Doping of the substrate with $\mathrm{Cr}$ would be another way to decrease the thermal conductivity.

Alternatively, we estimate the temperature profile using literature values of the thermal conductivity $\kappa$. From Ref. [16], we deduce a $\kappa$ of about $0.01 \mathrm{~W} / \mathrm{mK}$ at $300 \mathrm{mK}$. Using the simulation software COMSOL, a temperature difference of $2.5 \mathrm{mK}$ between the center and edge of our sample is found from the heat-flow equation. This would lead to a temperature difference across ring 2 of about $1 \mathrm{mK}$, in good agreement with the used fit values. 


\section{THERMOELECTRIC PERFORMANCE}

The quality of the thermoelectric performance, i.e., the efficiency (for a heat engine) or the coefficient of performance (for a refrigerator), has been the subject of great interest in recent years, particularly in the context of the ballistic transport along edge channels and nanosized devices [22-30]. We find that the theoretical performance of a Corbino device in the quantum Hall regime is surprisingly large, being comparable to the highest predicted values in devices based upon ballistic transport. The performance is parameterized as the figure of merit [20], $Z T=\mathcal{L}_{21}^{2} /$ Det $\hat{\mathcal{L}}$, or $S^{2}$ times the ratio of the electric and thermal conductivity.

The optimal Carnot efficiency or coefficient of performance is achieved for $Z T \rightarrow \infty$.

The highest reported values in real, usually semiconducting, materials are between $1 \leq Z T \leq 2.7$ [20,31], while optimistic theoretical predictions in the ballistic edge-channel regime are $Z T \sim 4$ [32] or lower. In Fig. 8 , we show the transmission function $\mathcal{T}(\varepsilon)$ used for the Corbino in this work to fit the experimental data of Fig. 3 within the high-magnetic-field regime. We see that the sequence of sharp features at the LL realizes energy filters, leading to large values of $Z T \sim 6$. Thus, diffusive transport across the bulk of a Corbino device has a potentially higher performance than the envisioned edge-channel devices. Figure 8 suggests that even higher $Z T$ values should be possible at lower temperatures. We stress that this analysis is based on the assumption that the main contribution to the thermoelectric and thermal transport is due to the electrons. Phononic thermal transport in the substrate would tend to decrease the performance but would die out at even lower temperatures with $T^{3}$, while the figure of merit would probably increase. Thus one could envision that the Corbino device could be used as a thermoelectric cooler in
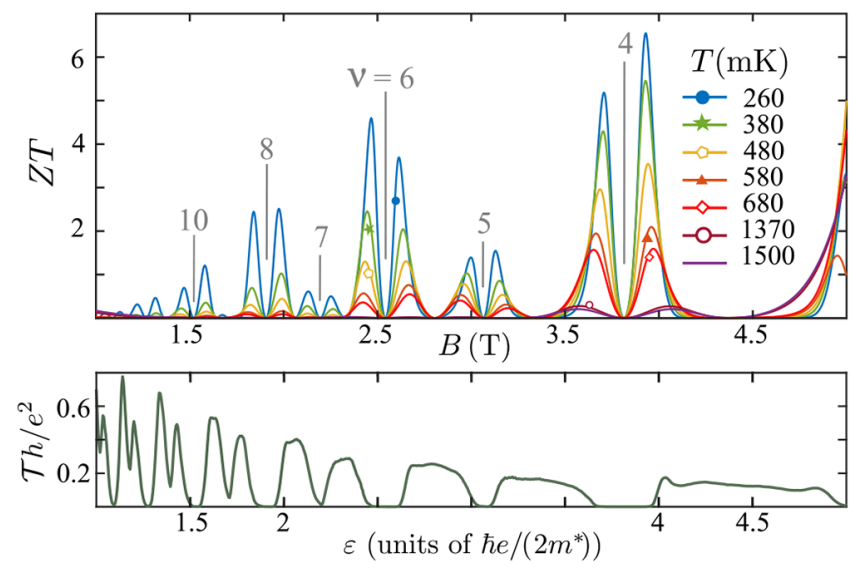

FIG. 8. Bottom: the transmission function $\mathcal{T}(\varepsilon)$. Top: the electron contribution to the figure of merit $Z T$. the low-millikelvin regime for specific purposes. Replacement of the heater by the object to be cooled could already be sufficient to form a realistic device.

\section{CONCLUSIONS}

We analyze the thermoelectric response of a Corbino structure in the quantum Hall regime. For partially filled LLs, we find excellent agreement between the experimental data and the theoretical description, based on the assumption that the thermoelectric response originates in the diffusion of electrons while electron-phonon drag does not influence the thermovoltage in the temperature range from $300 \mathrm{mK}$ to $2 \mathrm{~K}$. Clearly, the electron-phonon interaction does not vanish in the Corbino geometry but the transfer of momentum from the phonons to the electrons does not lead to a measurable voltage. Actually, it has already been noted a long time ago that the contribution of the phonon-drag mechanism to the thermoelectric coefficient $\mathcal{L}_{12}$ should be zero in the heat-flow direction [10], which is, simply put, a consequence of the Lorentz force. It appears that only in Corbino devices is the vanishing contribution of phonon drag reflected in the thermovoltage measurement. Within the diffusive model applicable for Corbino rings, we are able to accurately fit the temperature difference, producing the thermopower based on the measured conductance traces, and we find it to be consistent with both our experimental temperature estimates and that derived from independent thermal-conductivity data. The calculated figure of merit $Z T$ is remarkably high for high magnetic fields, indicating that this system is very promising as a low-temperature cooling device or a heat engine.

Future work needs to clarify the origin of the large voltage signals at the conductance minima, i.e., in the quantized state, where both the electric conductance and the thermal conductance values of the Onsager equation vanish. Also, different mechanisms might be relevant in these regimes, such as temperature-driven magnetic flux [13] or temperature-dependent contact potentials that cannot equilibrate in the conductance minima [33]. Another important direction would be the extension of the experiment to lower temperatures. Determination of the entropy in the fractional quantum Hall regime could answer some urgent questions about the entropy of the suspected non-Abelian states.

\section{ACKNOWLEDGMENTS}

We thank Klaus von Klitzing for his constant interest and support, Achim Güth and Marion Hagel for the wafer lithography, and Mirko Lupatini, Luca Alt, and Simon Parolo for help with the experiments. Peter Märki provided the amplifiers used in this work, while Lars Tiemann contributed the measurement software NANOMEAS. We received useful comments on the manuscript from 
Peter Samuelsson. We acknowledge support from the Instituto Nacional de Tecnología Industrial (INTI) and the National Scientific and Technical Research Council (Consejo Nacional de Investigaciones Cientficas y Técnicas, CONICET), Argentina. We are sponsored by Grant No. PIP-RD 20141216-4905 of CONICET, Grants No. PICT-2017- 2726 and No. PICT-2018 from Argentina, the Swiss National Foundation (Schweizerischer Nationalfonds) National Center of Competence in Research (NCCR) "Quantum Science and Technology," as well as the Alexander von Humboldt Foundation, Germany.

[1] K. v. Klitzing, G. Dorda, and M. Pepper, New Method for High-Accuracy Determination of the Fine-Structure Constant Based on Quantized Hall Resistance, Phys. Rev. Lett. 45, 494 (1980).

[2] D. C. Tsui, H. L. Stormer, and A. C. Gossard, TwoDimensional Magnetotransport in the Extreme Quantum Limit, Phys. Rev. Lett. 48, 1559 (1982).

[3] R. B. Laughlin, Anomalous Quantum Hall Effect: An Incompressible Quantum Fluid with Fractionally Charged Excitations, Phys. Rev. Lett. 50, 1395 (1983).

[4] J. K. Jain, Microscopic theory of the fractional quantum Hall effect, Adv. Phys. 41, 105 (1992).

[5] T. Gerster, A. Müller, L. Freise, D. Reifert, D. Maradan, P. Hinze, T. Weimann, H. Marx, K. Pierz, H. W. Schumacher, F. Hohls, and N. Ubbelohde, Robust formation of quantum dots in $\mathrm{GaAs} / \mathrm{AlGaAs}$ heterostructures for single-electron metrology, Metrologia 56, 014002 (2018).

[6] C. Nayak, S. H. Simon, A. Stern, M. Freedman, and S. Das Sarma, Non-Abelian anyons and topological quantum computation, Rev. Mod. Phys. 80, 1083 (2008).

[7] K. Yang and B. I. Halperin, Thermopower as a possible probe of non-Abelian quasiparticle statistics in fractional quantum Hall liquids, Phys. Rev. B 79, 115317 (2009).

[8] Y. Barlas and K. Yang, Thermopower of quantum Hall states in Corbino geometry as a measure of quasiparticle entropy, Phys. Rev. B 85, 195107 (2012).

[9] R. Fletcher, M. D'Iorio, W. T. Moore, and R. Stoner, A search for trends in the thermopower of GaAs- $\mathrm{Ga}_{1-x} \mathrm{Al}_{x} \mathrm{As}$ heterojunctions, J. Phys. C 21, 2681 (1988).

[10] T. M. Fromhold, P. N. Butcher, G. Qin, B. G. Mulimani, J. P. Oxley, and B. L. Gallagher, Phonon-drag magnetothermopower oscillations in $\mathrm{GaAs} / \mathrm{As}_{x} \mathrm{Ga}_{1}-x$ As heterojunctions, Phys. Rev. B 48, 5326 (1993).

[11] B. Tieke, U. Zeitler, R. Fletcher, S. A. J. Wiegers, A. K. Geim, J. C. Maan, and M. Henini, Even-Denominator Filling Factors in the Thermoelectric Power of a TwoDimensional Electron Gas, Phys. Rev. Lett. 76, 3630 (1996).

[12] S. Maximov, M. Gbordzoe, H. Buhmann, L. W. Molenkamp, and D. Reuter, Low-field diffusion magnetothermopower of a high-mobility two-dimensional electron gas, Phys. Rev. B 70, 121308 (2004).

[13] V. T. Dolgopolov, A. A. Shashkin, N. B. Zhitenev, S. I. Dorozhkin, and K. von Klitzing, Quantum Hall effect in the absence of edge currents, Phys. Rev. B 46, 12560 (1992).
[14] H. van Zalinge, R. W. van der Heijden, and J. H. Wolter, Anisotropic Corbino magnetothermopower in a quantum Hall system, Phys. Rev. B 67, 165311 (2003).

[15] S. Kobayakawa, A. Endo, and Y. Iye, Diffusion thermopower of quantum Hall states measured in Corbino geometry, J. Phys. Soc. Jpn. 82, 053702 (2013).

[16] W. E. Chickering, J. P. Eisenstein, L. N. Pfeiffer, and K. W. West, Thermopower of two-dimensional electrons at filling factors $v=3 / 2$ and 5/2, Phys. Rev. B 81, 245319 (2010).

[17] W. E. Chickering, J. P. Eisenstein, L. N. Pfeiffer, and K. W. West, Thermoelectric response of fractional quantized Hall and reentrant insulating states in the $N=1$ Landau level, Phys. Rev. B 87, 075302 (2013).

[18] J. P. Wolfe, Imaging Phonons: Acoustic Wave Propagation in Solids (Cambridge University Press, Cambridge, 1998).

[19] P. Märki, B. A. Braem, and T. Ihn, Temperature-stabilized differential amplifier for low-noise dc measurements, Rev. Sci. Instrum. 88, 085106 (2017).

[20] G. Benenti, G. Casati, K. Saito, and R. S. Whitney, Fundamental aspects of steady-state conversion of heat to work at the nanoscale, Phys. Rep. 694, 1 (2017).

[21] M. Jonson and S. M. Girvin, Thermoelectric effect in a weakly disordered inversion layer subject to a quantizing magnetic field, Phys. Rev. B 29, 1939 (1984).

[22] R. Sánchez, B. Sothmann, and A. N. Jordan, Chiral Thermoelectrics with Quantum Hall Edge States, Phys. Rev. Lett. 114, 146801 (2015).

[23] S. Kheradsoud, N. Dashti, M. Misiorny, P. P. Potts, J. Splettstoesser, and P. Samuelsson, Power, efficiency and fluctuations in a quantum point contact as steady-state thermoelectric heat engine, Entropy 21, 777 (2019).

[24] D. Gresta, M. Real, and L. Arrachea, Optimal Thermoelectricity with Quantum Spin Hall Edge States, Phys. Rev. Lett. 123, 186801 (2019).

[25] P. P. Hofer and B. Sothmann, Quantum heat engines based on electronic Mach-Zehnder interferometers, Phys. Rev. B 91, 195406 (2015).

[26] P. Samuelsson, S. Kheradsoud, and B. Sothmann, Optimal Quantum Interference Thermoelectric Heat Engine with Edge States, Phys. Rev. Lett. 118, 256801 (2017).

[27] L. Vannucci, F. Ronetti, G. Dolcetto, M. Carrega, and M. Sassetti, Interference-induced thermoelectric switching and heat rectification in quantum Hall junctions, Phys. Rev. B 92, 075446 (2015).

[28] P. Roura-Bas, L. Arrachea, and E. Fradkin, Enhanced thermoelectric response in the fractional quantum Hall effect, Phys. Rev. B 97, 081104(R) (2018).

[29] F. Giazotto, F. Taddei, M. Governale, R. Fazio, and F. Beltram, Landau cooling in metal? Semiconductor nanostructures, New J. Phys. 9, 439 (2007).

[30] L. Fu, Cryogenic Cooling and Power Generation Using Quantum Hall Systems, arXiv:1909.09506.

[31] J. He and T. M. Tritt, Advances in thermoelectric materials research: Looking back and moving forward, Science 357, 6358 (2017).

[32] A. Ozaeta, P. Virtanen, F. S. Bergeret, and T. T. Heikkilä, Predicted Very Large Thermoelectric Effect in Ferromagnet-Superconductor Junctions in the Presence of a Spin-Splitting Magnetic Field, Phys. Rev. Lett. 112, 057001 (2014).

[33] K. von Klitzing, Private Communication. 